This is the authors' version of the journal paper:

Santos-Assunçao, S., Dimitriadis, K., Konstantakis, Y., Perez-Gracia, V., Anagnostopoulou, E., Gonzalez-Drigo, R. (2016). Ground-penetrating radar evaluation of the ancient Mycenaean monument Tholos Acharnon tomb. Near Surface Geophysics, 14(2), 197-205.

\title{
Ground penetrating radar evaluation of the ancient Mycenaean monument Tholos Acharnon tomb
}

S. Santos-Assunçao(1), K. Dimitriadis(2), Y. Konstantakis(2), V. Perez-Gracia (1), E. Anagnostopoulou(3), R. Gonzalez-Drigo(1)

(1) Department of Strength of Materials and Structural Engineering, EUETIB/CEIB, Polytechnic University of Catalonia, C/Urgell 187, 08036 Barcelona (Spain) sonia. assuncao@upc.edu; vega.perez@upc.edu; jose.ramon.gonzalez@upc.edu

(2) Geoservice, Geological \& Geophysical Consultants, 35, Lykaiou St, 11476 Athens, Greece,workst@geoservice.gr

(3) Second Ephorate of Prehistoric and Classical Antiquities, Greece

\section{$\underline{\text { Abstract }}$}

The assessment of cultural heritage requires high-resolution and non-destructive methodologies. Ground penetrating radar is widely applied in the inspection of historical buildings. However, some structures with curved surfaces make the radar data acquisition process difficult and consequently the following data interpretation. This paper describes a case study concerning a circular and buried Greek monument. This monument is a magnificent tomb buried with irregular stones. However, its structure and the internal stones arrangement are unknown. Therefore, a radar survey was carried out to achieve two main objectives: (1) Identification of hidden elements and arrangement of the stones and (2) Detection of specific zones where further restoration and maintenance should be recommended. The methodology for the radar data acquisition involves the use of a laser scan in order to define accurately each radar line, covering all the internal surface of the tomb. Radar data processing was developed converting Cartesian coordinates into polar coordinates. This procedure allows defining better the internals anomalies, improving the radar data interpretation. The main results of the survey were three: (a) the presence of a hidden target buried in the corridor access to the tomb; (b) the description of the internal structure of the walls of the tomb, defining the stones arrangement and the position and depth to the key- 
stone; and (c) the existence of delimited zones where the signal is highly attenuated, probably due to a high salt content.

Keywords: Ground Penetrating Radar, Cultural Heritage, Archaeology, NonDestructive Testing, polar coordinates, Mycenaean Civilization

\section{Introduction}

Historical monuments are irreplaceable and essential resources in the study of ancient societies and the human history. Greece contains a large number of valuable sites with construction remains of significant civilization. The technical inspection of those structures is fundamental to preserve their identities as historical heritage and to understand the infrastructures and development of the civilizations. Although, conserving or restoring ancient architectonic constructions is a difficult task: it is necessary to preserve their original appearance and to maintain the construction without risk of collapse, with minimum intervention. Therefore, the accurate documentation of the structure might be the first step in the conservation tasks. Different surveys and studies could be applied in order to collect this documentation. Modern topographic surveys allow obtaining precise three dimensional models of the structures (e.g., Arias et al., 2007; Perez-Gracia et al., 2011). Furthermore, the knowledge of the inner elements and constructive structures, as well as possible internal damage, is also decisive in restoration and conservation processes. The inner information is usually gathered by means of non-destructive surveys in many cases.

Ground Penetrating Radar (GPR) is a non-destructive geophysical surveying technique that is nowadays applied to structures assessment. The methodology lays in the emission of high frequency electromagnetic pulses that travel trough the medium. The changes in the electromagnetic properties of the medium are discontinuities, which are identified by the reflection of the energy. As consequence, the technique provides images where the depth to the discontinuity is determined by the two-way travel time of the reflected waves on the A-Scans. The projection on the surface is defined by the coordinates of the position of the receiver antenna in the B-Scans. The knowledge of the depth and position of buried targets is fundamental in archaeological applications. Hence, the method has been widely used in this field during the last decades (Neubauer et al., 2002; Conyers and Leckebusch, 2010, Trinks et al., 2010; PerezGracia et al., 2012). A complete review of GPR procedures in archaeology can be found in Conyers and Goodman (1997). .

Moreover, this methodology may provide information about the inner structure and about the existence of any possible damage or deformations that might affect the constructive elements of archaeological complexes or cultural heritage structures. This information is important in the assessment of constructive archaeological remains or cultural heritage, and radar applications have been performed in different cases, with the objective of defining those aspects in diverse structures. For example, Hruiska and 
Fuchs (1999) presented the study with GPR of the road structure in old Greek cities; Perez-Gracia et al. (2008) and Solla et al. (2011) described a GPR analysis of Roman monuments in Spain; Leucci et al. (2007) applied GPR to evaluate mediaeval columns and Calia et al. (2013) combined GPR with other non-destructive techniques to evaluate a mediaeval crypt; Pérez-Gracia et al. (2000) explained the survey in a gothic cathedral; Santos-Assunçao (2014) describe the complete analysis of art nouveau masonry buildings; Faize et al. (2012) employed a $2.3 \mathrm{GHz}$ antenna to detect anomalies on a buried marble and to create a pathological model. These studies highlight the ability of GPR high frequency antennas to identify small size cracks, voids and hidden members in ancient monuments, evidencing the inherent difficulty in the interpretation of data from masonry members (Santos-Assunçao et al., 2014).

Some of the ancient Greek Monuments still remain against the time keeping their glorious identity. Among these monuments are several tombs that had been constructed according to specific architecture characteristic of their epoch and, in many cases, were covered by a tumulus. Generally, the lack of periodic inspection and preservation of the ancient structures could increase the action of different damaging factors that could be diminished by implementing the adequate maintenance.

This paper presents the study of the Tholos Acharnon tomb, a Greek monument representing a valuable heritage of the Mycenaean culture. The tomb is located in northern part of the capital Athens, in the city of Acharnes (Fig. 1). Acharnes has been an important city since the old times.

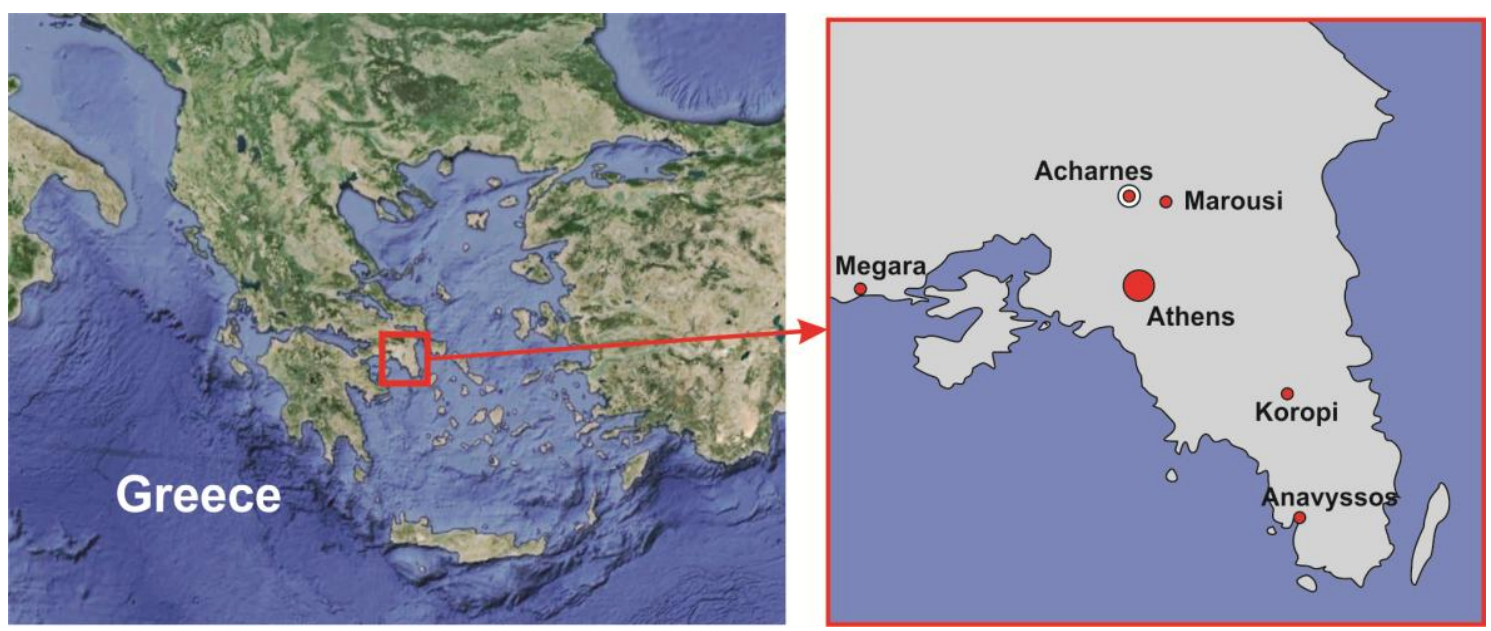

Figure 1 - Location of Acharnes, the site where the tomb is placed.

\section{The tomb of Acharnon}

The Mycenaean Tholos tomb ( $13^{\text {th }}$ cent. B.C.) at Acharnai (Menidi) was excavated by the German Archaeological Institute in 1879 (Camp, 2004). It is a subterranean structure mounded with earth sustained all around by a low wall. The descending corridor $(27,72 \mathrm{~m}$ long, $3 \mathrm{~m}$ wide) leads through an entrance passage $(3,55 \mathrm{~m}$ long, $1,55 \mathrm{~m}$ wide) to the burial chamber $(8,35 \mathrm{~m}$ diameter, $9 \mathrm{~m}$ approximately height). The 
floor of the chamber is nearly circular. It was built as a corbelled vault with walls tapering towards the apex, where the final opening is closed with a big slab. The chamber had the shape of a beehive. The usual relieving triangle above the lintel is visible only on the inner side of the tomb, while outside exterior a more original system was adopted by alternating hollows with four small stones placed horizontally (Fig. 2).

The Tholos tomb at Acharnai was built to receive the members of the elite family, most probably the family of the region governor.

Although the tomb was plundered, probably in antiquity, it contained many findings belonging to the dead person such as jewelry of gold, silver, bronze, electrum, glass paste and faience, seals of semi precious stones, bronze weapons, and a boar tusk helmet, in addition to a lot of ivory items like a cylindrical box in relief decoration of rams and fragments of two lyres, clay and stone vases like the clay amphora from Canaan with the last one denoting relations to southeastern Mediterranean. Findings in the corridor suggest that in later years until the $5^{\text {th }}$ cent. B.C. honor was attributed to the ancestors. Valuable findings (Fig. 2) are displayed in the National Archaeological Museum in Athens.


Figure 2. a) The tomb of Acharnon and the Dromos: the access from the stairs to the entrance.

b) Two examples of the archaeological findings in the tomb: a box of jewelry and an ivory lyre.

\section{Visual inspection: constructive materials, structure and deterioration}

A corridor is the access to the tomb that is buried under a tumulus. The tomb is built with irregular schist, limestone and sandstone materials. The outer wall is mainly constituted by schist and sandstone rocks. Some of these materials are remarkably porous or fissured (Fig. 3). Additionally, there is a lack of mortar between layers and elements, increasing the porosity and fragility of the whole structure. Inside of the beehive tomb, the materials are similar to those in the walls of the corridor, but 
pathologies are visible and damage seems to be more important. Visual inspection showed the existence of many voids between blocks of rock (Fig. 4).

The temperature oscillates significantly inside the tomb due to the seasonal changes characterizing the regional climate. However, the humidity is always high, increasing the vulnerability of the Monument. Moreover, due to the low temperatures in the winter season, in Acharnes it is usual to add salt in order to unfreeze the ice over the road close to the tomb. The salt that is dissolved in rainwater, seeps into the ground increasing the salinity of the medium around the tomb and, consequently, inside the ceiling and walls. The visual inspection highlights small salt deposits inside the tomb, in the upper part of the structure. Figure 4 shows several evidences of pathologies inside the tomb, such as the irregular setting of stones causing voids, oxidation of some materials and salt efflorescence on the blocks of rock.

A further cause of damage in the tomb structure could be the vibrations induced by the traffic. The adverse effects of long-term vibrations on historic buildings have been studied in several works (e.g., Rainer, 1982), because they are one of the factors that potentially reduce the timelife of structures. The monument is close to a high traffic road, and the effect of vibrations on the tomb must be taken into consideration. These vibrations could be the cause of detaching of some small stones from the upper part of the tomb structure and the fine grained material that appears in some zones, between the rock blocks. Additionally, some visual damages could be associated to stronger vibrations caused by the regional seismic activity.

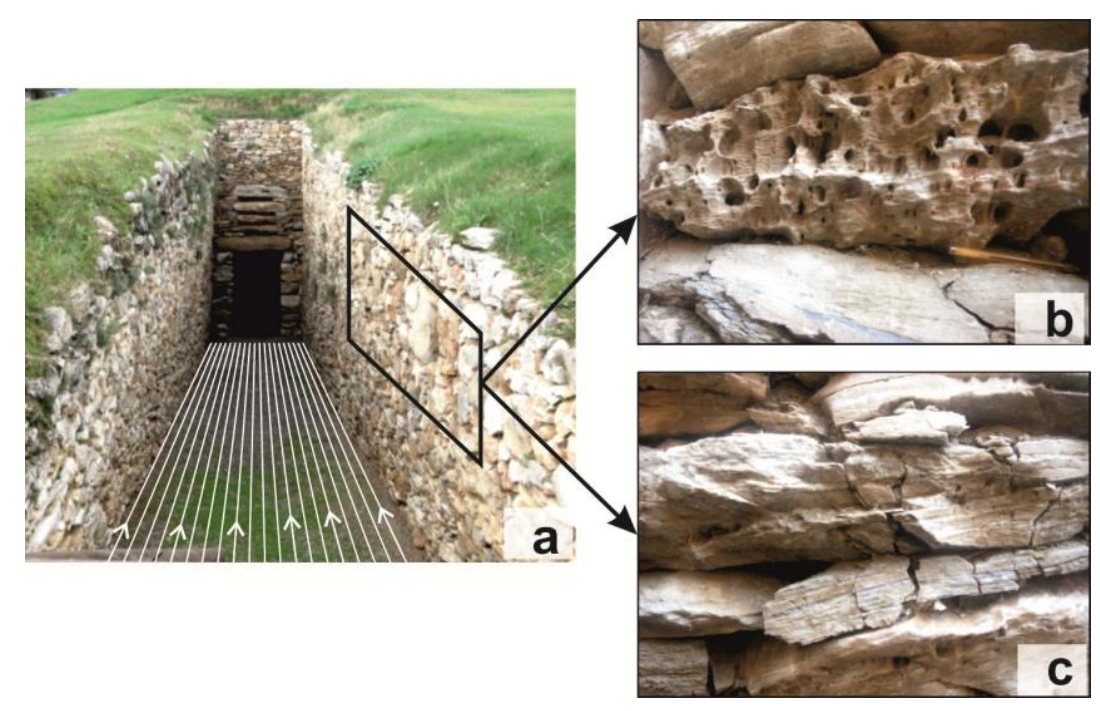

Figure 3- (a) Visual inspection in the walls of the access corridor with the GPR lines marked on the floor. (b) Porous and (c) fissured rocks are the most usual components of the structure. 


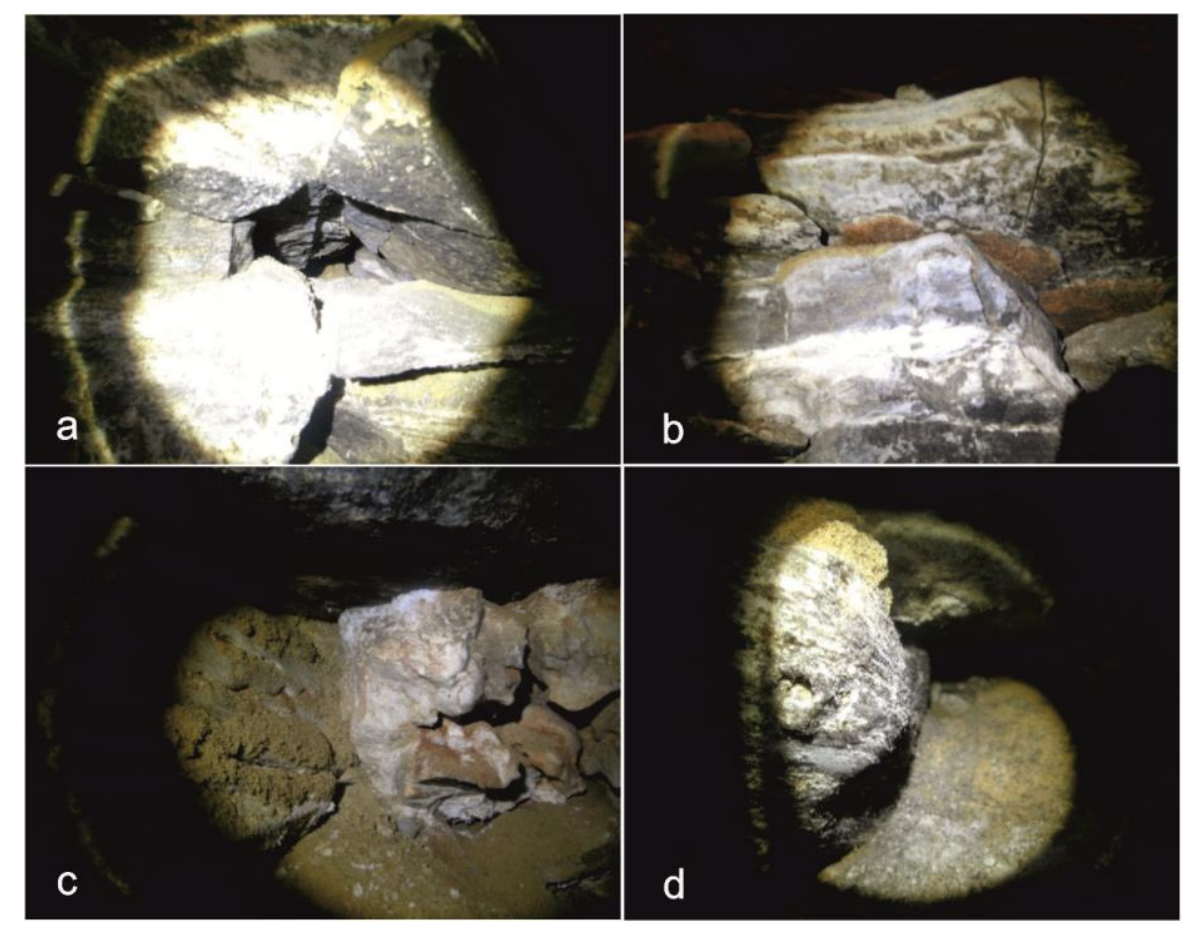

Figure 4 - Damage in the interior structure. a) Superposition rocks irregularly disposed is one of the causes of numerous voids inside the walls. b) Oxidation of materials. c) Existence of finegrained soil between the rocks. d) Salt efflorescence on the surface of the rocks.

\section{Ground-penetrating radar assessment}

Three parts of the tomb were assessed with GPR: the corridor (the Dromos), the interior of the tomb and the tumuli outside the tomb.

\section{The Dromos}

The Dromos is the access to the buried tomb. It is a long corridor with the floor covered with gravel. GPR survey in that corridor was focused on studying the subsoil. Radar data was acquired in 20 parallel profiles in the soil along the Dromos with an $800 \mathrm{MHz}$ centre frequency antenna. The distance between radar lines was $10 \mathrm{~cm}$. The objective of this study was to detect anomalies in the radar images that could determine the possible existence of buried archaeological findings.

Radar data acquisition parameters were selected after first tests in-situ, bearing in mind the objectives of the study. Hence, the distance between traces was $1.9 \mathrm{~cm}$, the time window was set to $50.3 \mathrm{~ns}$, and the sampling frequency to $10169 \mathrm{MHz}$. This parameter is the inverse of the sampling interval, the interval at which the incoming signals are read. Depending on the time window, the dielectric properties of the medium and the spectrum bandwidth, is recommended to set at about 10 times the antenna frequency. The number of samples for each A-scan was 512 . 


\section{The Tomb}

The main objective of the study of the interior of the tomb was to define the number of stone layers of the structure, and evaluate also their state and condition. However, the prospection inside the tomb with the GPR was a complicated task due to the irregular distribution of stones in the walls, including voids and discontinuities. Moreover, radar data acquisition was also difficult as consequence of the shape and size of the structure. Therefore, a previous experimental step was carried out before accomplishing the complete survey inside the tomb, with two main purposes:

(1) Determine the metric precision of the survey wheel over such irregular surface and compare the results obtained with the $1 \mathrm{GHz}$ and the $2.3 \mathrm{GHz}$ centre frequency antennas, evaluating penetration depth and intensity of the scattering in the radar images.

(2) Estimate the average wave velocity. This test was done by conducting several GPR measurements over well-defined and depth-known objects inside the tomb, in addition to the inserting of an aluminum plate at certain distances (depths) between the stone blocks (Fig. 5) and picking its reflections. This step provided an average estimation for the wave velocity of $10 \mathrm{~cm} / \mathrm{ns}$.

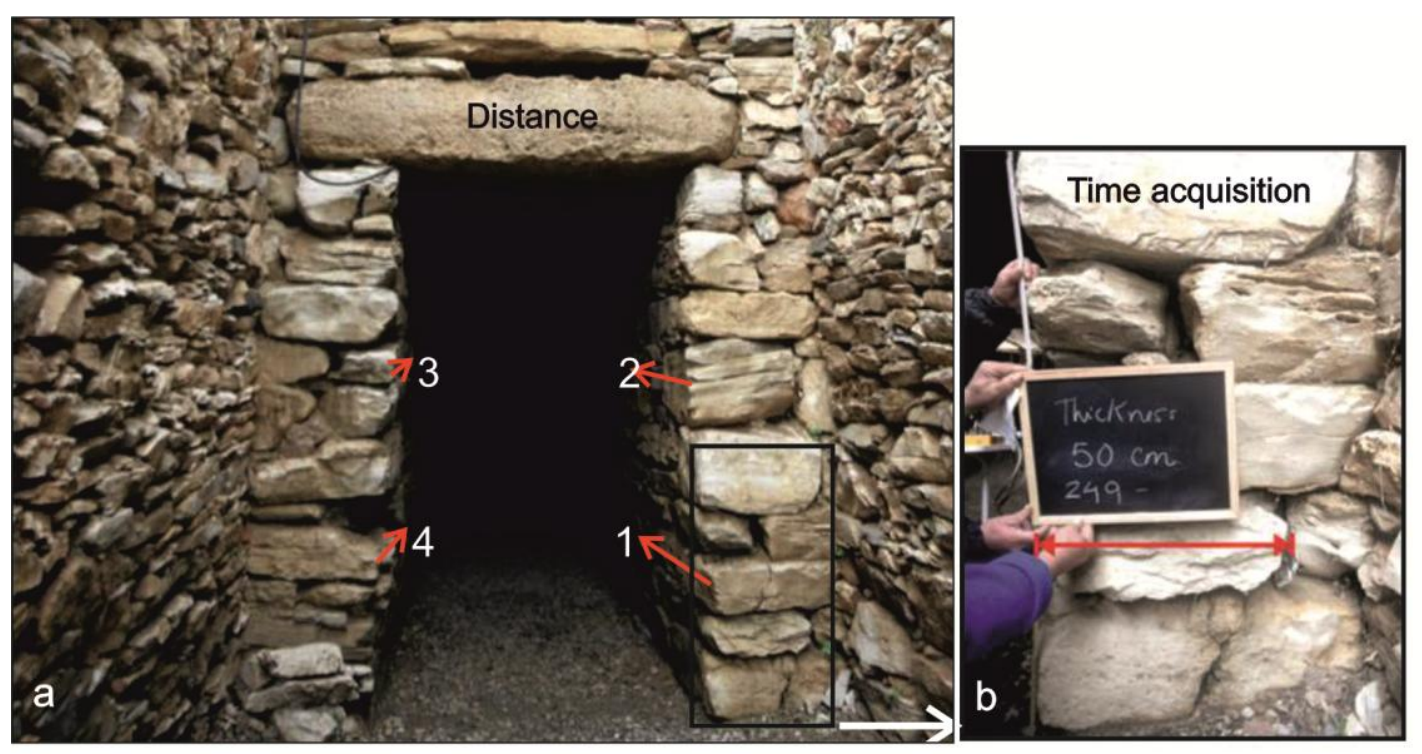

Figure 5. Previous measurements on the entrance of the Tomb in order to calibrate the GPR survey. a) Radar lines on the walls. b) Using an aluminum target to determine the wave velocity.

The first objective of the study of the interior walls of the tomb was to investigate the structure of the walls, defining the number of stone layers and detecting other possible hidden elements. The second objective was to detect important voids, cracks and any further elements usable for the assessment and restoration of the monument. 
Due to the height and the shape of the tomb, the survey required the use of scaffolding. The data acquisition was performed over three levels, as the scaffolding platform was assembled at three different heights.

The surface of the inner wall is composed of stones of diverse geological materials with irregular shapes. The radar data acquisition was tricky so that it was difficult to draw and follow a regular line path with the antenna on such rough surface. The irregularity of the line path changes the directivity of the antenna emission. Therefore, a laser scanning was used to assure the accuracy of the line relocation. The scanner was placed on each level of the scaffolding. The horizontal level was assured by means of a bubble level. The scanner was rotating the laser beam, drawing a circular line at a certain height where the antenna was moving along to form a measuring line (profile). Each profile was defined in the same way with a line marked by the laser scanner beam. The oscillation of the antenna was prevented by placing a thin layer of polyurethane on the wall surface, and moving the antenna on that layer (Fig. 6).

The roof of the Tomb has approximately a pseudo-conical form, with a decreasing radius at higher levels, and showing a particular beehive shape. The tomb is a circular buried structure, and the radius of each one of the radar lines was determined by topography. The radius is different for each height; as the height increases, the radius decreases. The value of the radius at each height corresponding to each radar line was introduced into the software processing (GPRSlice, 2014). A specific algorithm, recently developed, used this radius to warp the original radargram in order to show a circular pattern and provide a more realistic image. This algorithm processes the data accounting for the curvature of the interface. As a result, it is possible to obtain radargrams in polar coordinates, based on the original radargrams.

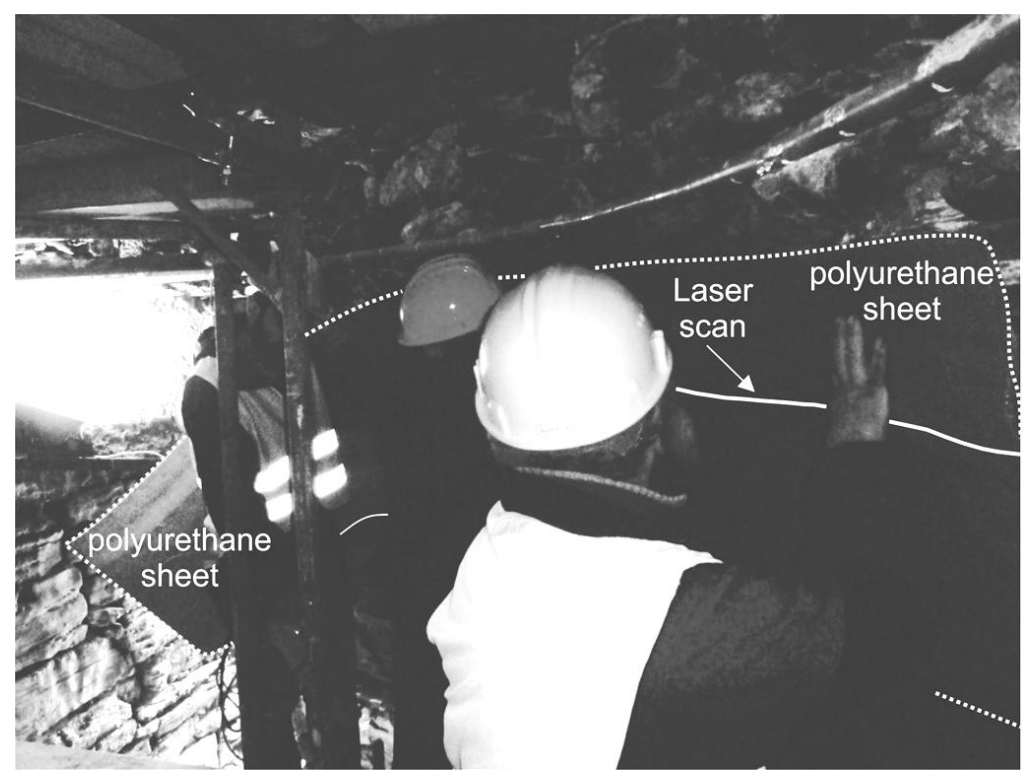

Figure 6 - Radar data acquisition inside of the Tomb. A laser scan marks the line of the radar profile on the polyurethane sheet. The antenna was moved over that sheet. 


\section{The tumulus outside the tomb}

The tomb is a subterranean structure buried under the tumulus. The tumulus materials were evaluated by means of the $500 \mathrm{MHz}$ centre frequency antenna. This part of the survey was oriented to determine zones with altered soil and to detect the keystone of the tomb, defining its exact position under the soil. The keystone, also called headstone, is the central wedge-shaped stone of the domed structure of the tomb that locks its parts together, being the central supporting element.

The shape of the tumulus (Fig. 7) makes the topographic corrections necessary for radar data, in order to determine accurately the depth to of the targets.

An aluminum square plate with sides of $40 \mathrm{~cm}$ was placed on the keystone from the tomb's inner side to enhance the wave reflectivity. The energy reflected on the metal increased the detectability of the keystone producing a higher contrast of the anomaly in the radargram.

Several profiles were distributed over the area (Fig. 7). One of the radar lines crosses the higher part of the tumulus where the keystone was detected.
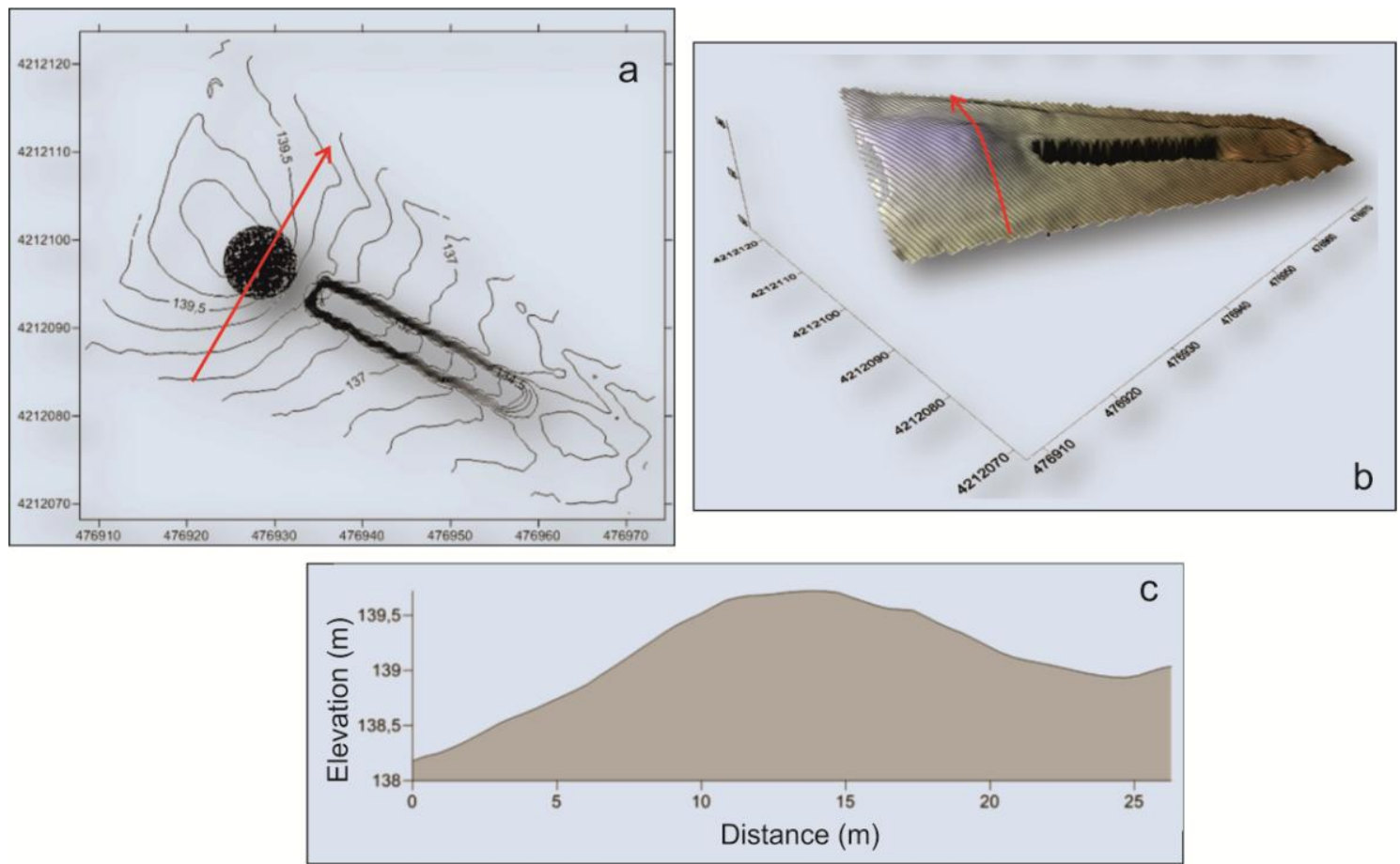

Figure 7-Topography of the tumulus. a) Topographic results and one radar line (red arrow). b) 3D topographic reconstruction of the tumulus and the tomb entrance. The arrow represents the radar line used to determine the position of the key stone of the structure. c) Topographic elevation along the radar line. 


\section{$\underline{\text { 5. Results }}$}

\section{The Dromos}

The collected data over the Dromos floor has been processed and presented in B-scan visualization. The processing flow included background removal, band pass filter between $400 \mathrm{MHz}$ and $1200 \mathrm{MHz}$ and manual gain. An example for the B-scan is given in Figure 8. The B-scan sections have been employed to construct 3D pseudo radar images and the corresponding slices (Fig. 9).

Reading constructed B-scans and 3D radar images for the floor of the Dromos indicates the existence of a visible interface between two shallow media, most likely the interface between the superficial gravel and the natural soil that is mainly composed of sand. This interface appears at about 2 ns depth (Fig. 8). Underneath this interface, a clearly defined anomaly is detected at $5 \mathrm{~ns}$. At about $10 \mathrm{~ns}$ depth it is possible to define a second interface between two different media. Underneath this depth, high attenuation of the signal impedes the analysis of deeper zones. This effect could be caused by higher clay or salt content in the ground. Furthermore, at 3.5 ns depth approximately, an anomaly related to a high amplitude signal was detected. The render isoamplitudes allowed selecting and defining the spatial limits of the anomaly, recognizing the extension and the shape of the reflective targets.
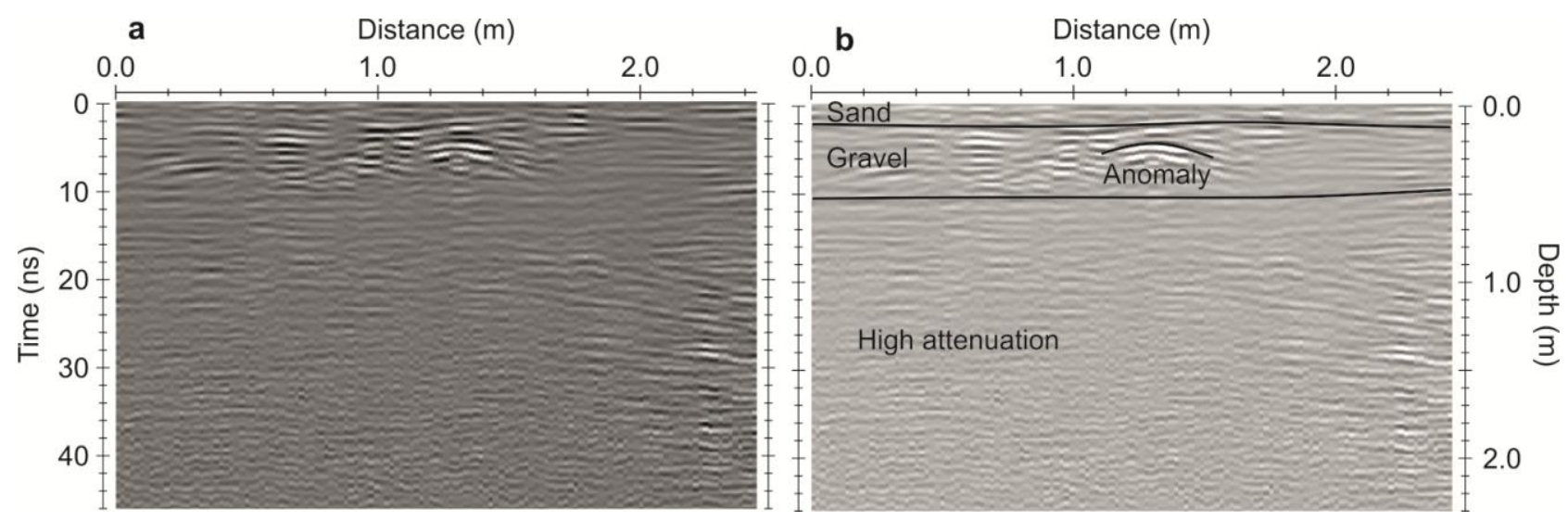

Figure 8. Radar data acquired in the Dromos. a) Processed B-scan. b) Possible radar data interpretation showing two ground interfaces and a visible anomaly at $5 \mathrm{~ns}$ depth. 


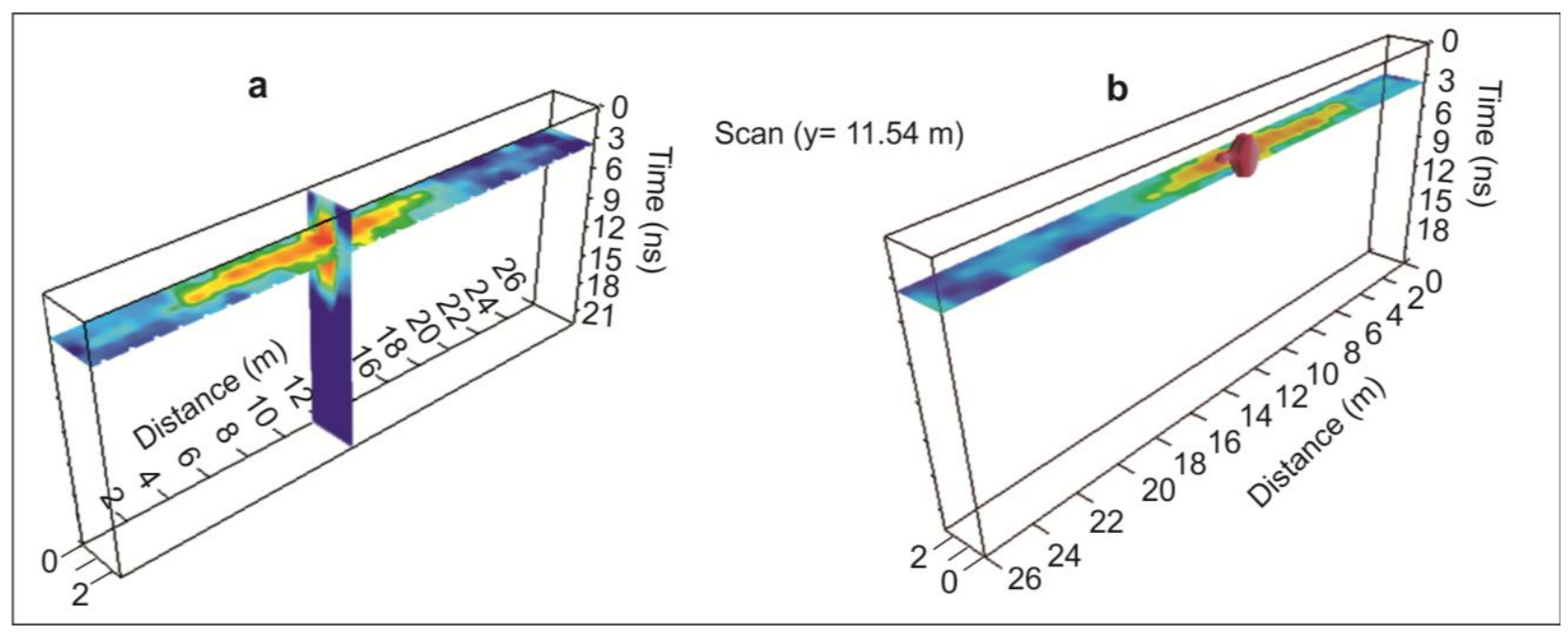

Figure 9. a) Horizontal and vertical cross sections intersected to highlight the target detected at $3.2 \mathrm{~ns}$ depth in the Dromos. b) Render isoamplitudes superimposed to the GPR slice provide an image that defines the possible shape of the target.

\section{The Tomb}

The tomb is a circular structure. Radar data was acquired along profiles, covering the entire inner side of the tomb. Cartesian coordinates were converted into polar coordinates warping the B-scans regarding the inner topography of the tomb. Figure 10 shows the radar image corresponding to one perimeter radar line, prior to coordinates transformation. The anomalies suggest the existence of a discontinuity inside the walls that could be caused by two irregular layers of stones. The result is a possible evidence of the tomb structure, heretofore unknown.

The circular images analysis allows also defining the existence of two possible irregular layers of stones. Figure 11 shows an example of one of the radar images. The geometry of the Tomb wall was considered and represented in radar data according to accurate topographic measurements.

Radar images in Figures 10 and 11 are characterized by a more regular anomaly that could be associated to an interface between two layers of stones, irregular anomalies as consequence of reflections on small targets and many small hyperbolas and discontinuities most likely associated to cracks, fissures and voids. The analysis of the wave velocity propagation inside the wall provided the information about the thickness of stone layers. The first layer of stones is approximately $25 \mathrm{~cm}$ thick, and a second layer is detected at about $50 \mathrm{~cm}$. The natural soil appears beneath these layers. These two thicknesses are average values since the stones layers are irregular due to the highly variable size and shape of the stones. 
Other interesting result is the high attenuation of the radar signal, observed in several areas. A possible cause of this effect is the presence of salt in the medium. The visual inspection identified salt efflorescence in different zones of the structure, as well as small salt deposits, corroborating this hypothesis. The GPR results facilitated defining and mapping the zones that could be affected with higher salt content.

Distance $(m)$

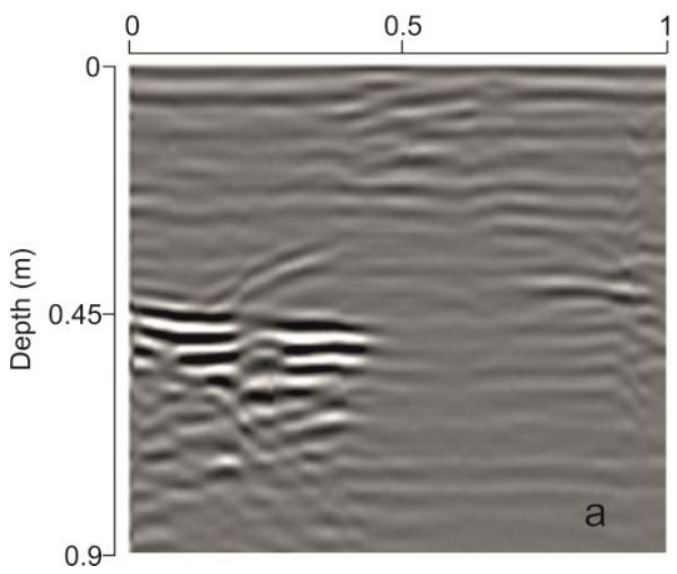

Distance (m)

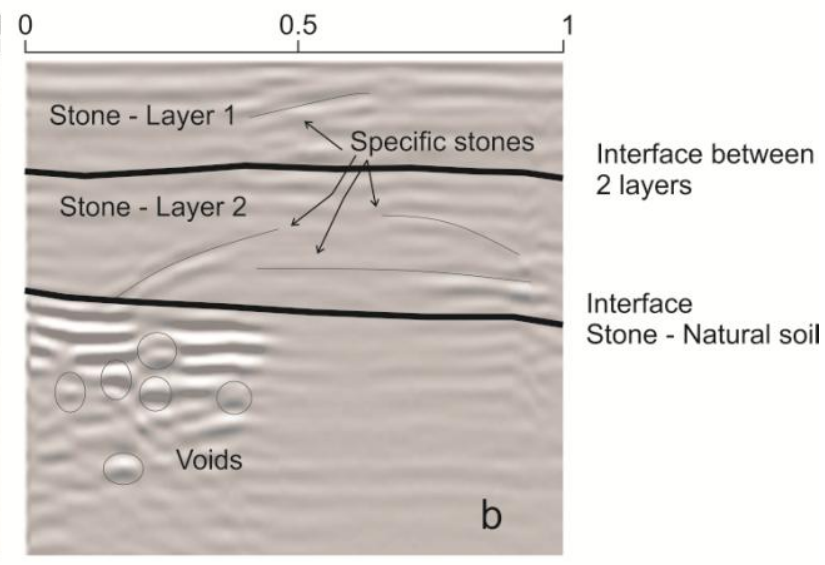

Figure 10. Radar data from part of the line placed at $23 \mathrm{~cm}$ high over the walls of the tomb. a) Processed radargram. b) Possible radar data interpretation. The image highlights the anomaly associated to the discontinuity between the two layers of stones, and the possible interface between the tomb structure and the natural soil. Several small hyperboles underneath the deeper interface are related to voids between the stones.
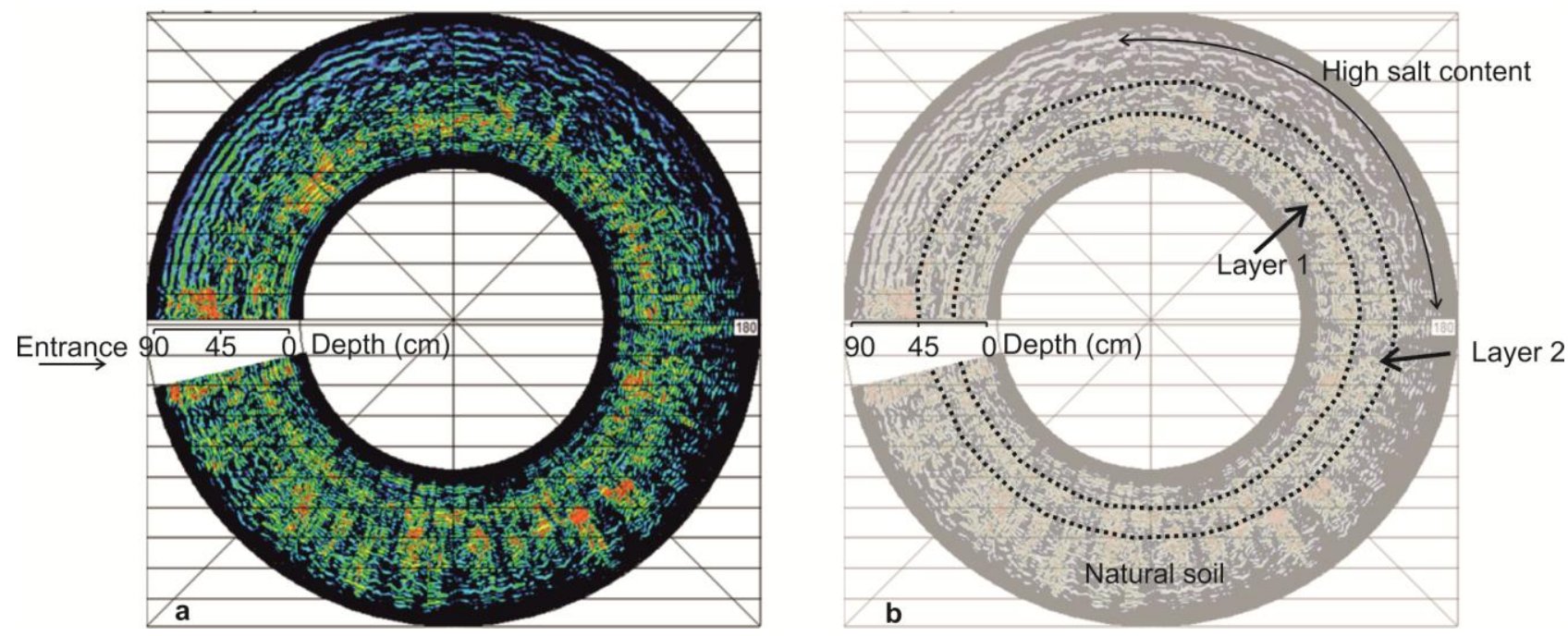

Figure 11. The entire radargram from the radar line placed at $23 \mathrm{~cm}$ high, after the coordinate transformation. a) Processed image, adapted to the tomb geometry. b) Possible radar data interpretation. Two layers are evidenced in the image. The zone corresponding to high signal attenuation is also defined. 


\section{The tumulus}

The main objectives of the survey on the tumulus were to define possible geological structures, and to detect the key stone of the tomb structure. Figure 12 shows part of the radar data acquired in the profile crossing the tumulus. Background removal, manual gain and subtract dewow filters have been applied in order to optimize the raw data image.

An evident anomaly in the radar image reveals the possible location of the keystone, at a depth of about $1 \mathrm{~m}$. Several hyperbolas in the upper part could be related to small stones that were even observed at the surface.
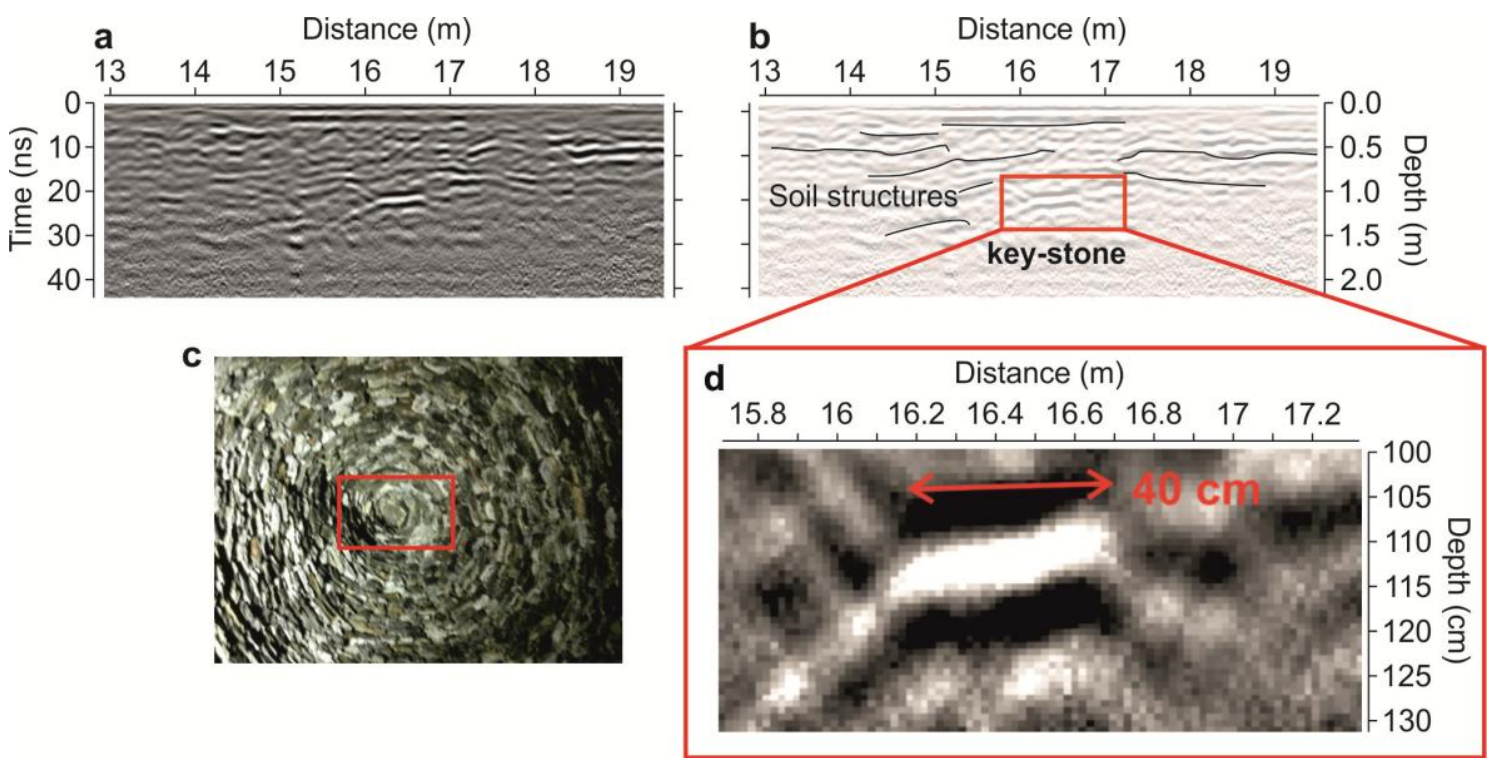

Figure 12. a) Radar data from the tumulus detecting the anomaly associated to the keystone. b) radar data interpretation. c) Photograph of the keystone, inside the tomb. d) Detail of the anomaly caused by the reflection on the keystone.

\section{Conclusions}

This paper presented a case study describing the extensive assessment of the Tomb of Acharnon. The survey covered the different parts of the monument: inside the tomb, the Dromos and the tumulus. The results underscore the ability of GPR for the inspection of irregular surfaces. High resolution images of the curved structure are obtained in order to define structural characteristics of the tomb and to detect the possible buried targets.

The investigation of the Dromos allowed distinguishing an anomaly characterized by high amplitude, perfectly defined in the space. The render isoamplitudes described a well-defined target, showing its extension and shape. The exact position of the target determined the area to be explorer with further testing. 
The assessment of the inner structure of the tomb determined the most characteristic features of the structure: the tomb was built by using two layers of irregular stones. The knowledge of this detail is of great interest for future structural studies and surveys oriented to projects of restoration, retrofitting and refurbishing. The discontinuities detected by means of GPR are located at a depth of 25 and $50 \mathrm{~cm}$, being most likely the first one the interface between the two layers of stones. The second interface probably corresponds to the contact between the buried structure and the natural soil. Radar images on polar coordinates defined an irregular first contact, associated to nonhomogeneous shaped stones.

The GPR study defined another important aspect that may be of interest to the conservation of the monument: some of the areas close to the tomb and also affecting zones of its walls caused a significant attenuation of the radar signal. This result was complemented with the visual inspection, concluding that there may be areas of high salinity in the subsoil, in the vicinity and around to the tomb. GPR analysis allows detecting the parts of the tomb affected by this problem. Moreover, the inspection of the tumulus and the surrounded area defines zones close to the tomb that must be controlled and considered in future conservation and restoration tasks that may be performed on the monument. The most important signal attenuation was observed near to the apex of the structure, which is an area situated in the vicinity of a road where the salt has been usually added to unfreeze the ice during the winter.

The inspection of the surrounded area and the tumulus allowed determining the exact location and depth of the key-stone.

All the information provided by the GPR inspection allowed understating the structural arrangement of the beehive structure of the tomb and detecting possible pathologies and zones which should require a special attention. The radar data processing in polar coordinates, defining better the shape of the structure, determined with accuracy the position of the anomalies and zones of interest.

\section{Acknowledgments}

This work has been partially funded by the EU funded COST Action TU1208, "Civil Engineering Applications of Ground Penetrating Radar" through the STSM "The preservation of the Tholos Tomb of Acharnon", and by the Spanish Government and the European Commission with FEDER funds, through the research project CGL201123621.

\section{References}

Arias P., Armesto J., Di-Capua D., Gonzalez-Drigo R., Lorenzo H., Perez-Gracia V., 2007, Digital photogrammetry, GPR and computational analysis of structural damages in a mediaeval bridge, Engineering Failure Analysis 14, 1444-1457. 
Calia A., Lettieri M., Leucci G., Matera L., Persico R., Sileo M., 2007, Journal of Archaeological Science 40, 4162-4169

Camp, J.M., The Archaeology of Athens, Yale University Press, London 2004, 340 pp.

Conyers L., Goodman D., Ground-Penetrating Radar: An Introduction for Archaeologists. Altamira Press, Walnut Creek, CA., 1997, 232 pp.

Conyers L., Leckebusch J., 2010, Geophysical archaeology research agendas for the future: some ground-penetrating radar examples, Archaeological Prospection 17, 117123.

Faize, A. and Driouach, A., 2012, The use of ground penetrating radar for the detection and study of a buried marble and in situ location of possible cracks, International Journal of Engineering Research and Applications 2(4), 1036-1039.

Geophysical Geometry Laboratory, 2014. GPR-SLICE V7.0 Ground Penetrating Radar Imaging Software User's Manual, CA, USA.

Hertrich M., Braun M., Yaramanci U., 2005, Magnetic resonance soundings with separated transmitter and receiver loops, Near Surface Geophysics 3, 141-154.

Hruska J., Fuchs G., 1999, GPR prospection in ancient Ephesos, Journal of Applied Geophysics 41, 293-312

Leucci G., Cataldo R., De Nunzio G., 2007, Assessment of fractures in some columns inside the crypt of the Cattedrale di Otranto using integrated geophysical methods, Journal of Archaeological Science 34, 222-232

Neubauer W., Eder-Hinterleitner A., Seren S., Melichar P., 2002, Georadar in the roman civil town carnuntum, austria: an approach for archaeological interpretation of GPR data, Archaeological Prospection 9, 135-156

Perez-Gracia V., Canas J.A., Pujades L.G., Clapes J., Caselles O., García F., Osorio R., 2000, GPR survey to confirm the location of ancient structures under the Valencian Cathedral (Spain), Journal of Applied Geophysics 43, 167-174

Perez-Gracia V., Di Capua D., Caselles O., Rial F., Lorenzo H., González-Drigo R., Armesto J., 2011 Characterization of a Romanesque Bridge in Galicia (Spain), International Journal of Architectural Heritage 5(3), $251-263$

Pérez-Gracia V., García F., Pujades L.G., González-Drigo R., Di Capua D., 2008, GPR survey to study the restoration of a Roman monument, Journal of Cultural Heritage 9, 89-96.

Perez-Gracia V., González-Drigo R., Sala R., 2012, Ground-penetrating radar resolution in cultural heritage applications, Near Surface Geophysics 10, 77-87 
Rainer J.H., 1982, Effect of Vibrations on Historic Buildings: An Overview, Bulletin of the Association for Preservation Technology 14(1), 2-10

Santos-Assunçao S., 2014. Ground Penetrating Radar Applications in Seismic Zonation: Assessment and Evaluation. PhD thesis. Polytechnic University of Catalonia, Barcelona-Tech.

Santos-Assunçao S., Perez-Gracia V., Caselles O., Clapes J., Salinas V., 2014, Assessment of complex masonry structures with GPR compared to other nondestructive testing studies, Remote Sensing 6, 8220-8237

Solla M., Lorenzo H., Rial F.I., Novo A., 2011, GPR evaluation of the Roman masonry arch bridge of Lugo (Spain), NDT\&E International 44, 8-12

Trinks I., Johansson B., Gustafsson J., Emilsson J., Friborg J., Gustafsson C., Nissen J., Hinterleitner A., 2010, Efficient, large-scale archaeological prospection using a true three-dimensional ground-penetrating Radar Array system, Archaeological Prospection $17(3), 175-186$ 